\title{
Low-lying magnetic loops in the solar internetwork
}

\author{
M. J. Martínez González ${ }^{1,2}$, M. Collados², B. Ruiz Cobo ${ }^{2}$, and S. K. Solanki ${ }^{3}$ \\ ${ }^{1}$ LERMA, Observatoire de Paris-Meudon, 5 place Jules Janssen, 92195 Meudon, France \\ e-mail: Marian.Martinez@obspm. fr \\ 2 Instituto de Astrofísica de Canarias, vía Láctea S/N, 31200 La Laguna, Spain \\ e-mail: mcv@iac.es, brc@iac.es \\ 3 Max-Planck-Institut für Sonnensystemforschung, Max-Planck-Str. 2, 37191 Katlenburg-Lindau, Germany \\ e-mail: solanki@mps.mpg.de
}

Received 19 March 2007 / Accepted 9 May 2007

\section{ABSTRACT}

\begin{abstract}
Aims. We study the structure of the magnetic field vector in the internetwork and search for the presence of small-scale loops. Methods. We invert $1.56 \mu \mathrm{m}$ spectropolarimetric observations of internetwork regions at disc centre by applying the SIR code. This allows us to recover the atmospheric parameters that play a role in the formation of these spectral lines. We are mainly interested in the structure of the magnetic field vector.

Results. We find that many opposite polarity elements of the internetwork are connected by short (2-6"), low-lying (photospheric) loops. These loops connect at least the 10-20\% of the internetwork flux visible in our data. Also we have some evidence that points towards a dynamic scenario that can be produced by the emergence of internetwork magnetic flux.
\end{abstract}

Key words. magnetic fields - Sun: atmosphere

\section{Introduction}

Magnetic fields appear on the solar surface in the form of bipolar regions, with the two polarities connected by magnetic field lines in the shape of loops. If the loops are sufficiently large they are traced in $\mathrm{H} \alpha$ (arch filament systems) or EUV or X-ray images (transition region and coronal loops). There is also mounting evidence for the presence of many small loop-like structures in the upper quiet atmosphere (e.g. Feldman et al. 1999) and in the photosphere (Lites et al. 1996; de Pontieu 2002), and the presence of such loops has been invoked by the complex mixture of magnetic polarities typical of the quiet Sun. Direct measurements of the full magnetic structure of a loop are extremely rare, being restricted to chromospheric loops in emerging flux regions (Solanki et al. 2003). Here we trace the field of low-lying loops and present evidence that they are common in the internetwork quiet Sun.

Our knowledge of the magnetic field in the internetwork has evolved rapidly in recent years. The magnetic features present in internetwork regions, as seen by the Zeeman effect, are unresolved, occupy a very small portion of the resolution element $(1-2 \%)$ and have field strengths of a few hundred $\mathrm{G}$ (Keller et al. 1994; Lin 1995; Lin \& Rimmele 1999; Khomenko et al. 2003; López Ariste et al. 2006b; Martínez González et al. 2007; Asensio Ramos et al. 2007a). The rest of the resolution element is likely filled by magnetic fields that currently are only detectable by means of the Hanle effect (Stenflo 1987) or the Zeeman-sensitive Mn I line at $1.52 \mu \mathrm{m}$ (Asensio Ramos et al. 2007a). If we assume that such a hidden magnetic field is tangled at subresolution scales and that it occupies the whole resolution element, the most sophisticated Hanle effect determinations measure a mean magnetic field of $100 \mathrm{G}$ (Trujillo Bueno et al. 2004) while previous estimates give a much weaker field
(Faurobert et al. 2001). In this type of study, the spatial resolution is given by the slit length, since the signal is averaged over the whole slit in order to obtain an adequate signal to noise ratio. In addition, some works find empirical evidence of this tangled nature (Stenflo 1987; Manso Sainz et al. 2004; Asensio Ramos et al. 2007a). The present paper extends the knowledge of the quiet Sun by revealing the connectivity of magnetic features in the internetwork and partly its three dimensional structure.

\section{Observations and data reduction}

The observational data consist of two scans of quiet Sun regions. One of them was located at disc centre and the other one at $\mu=0.88$. They were observed on the 29 th and the 30th of July 2000, respectively. All four Stokes parameters of the pair of Fe I lines at $1.56 \mu \mathrm{m}$ were recorded using the Tenerife Infrared Polarimeter (Collados 1999) installed at the Vacuum Tower Telescope at El Teide observatory. In both cases, the integration time at each slit position was $1 \mathrm{~min}$ which allowed us to achieve a noise level in the polarization profiles of $4.1 \times$ $10^{-4} I_{\mathrm{c}}$ in the observation at disc centre and $2.5 \times 10^{-4} I_{\mathrm{c}}$ in the one at $\mu=0.88$. Here, $I_{\mathrm{c}}$ is the continuum intensity. In Fig. 1 , the $I_{\mathrm{c}}$ maps are shown for both scanned areas. The slit was oriented vertically and scanned horizontally. We used a correlation tracker (Ballesteros et al. 1996; Schmidt \& Kentischer 1995) to correct for image motion due to the seeing. The continuum intensity contrast of both data sets is $1.4 \%$ and $2.0 \%$, respectively, for the maps at $\mu=0.88$ and $\mu=1$. In both maps, the granulation pattern is clear and the spatial resolution was estimated to be of the order of $1^{\prime \prime}$. This was computed using the Fourier transform of the $I_{\mathrm{c}}$ images and choosing the frequency where the power was 10 times above noise. In both cases the pixel size, the slit width and the scan step were all $0.4^{\prime \prime}$. 

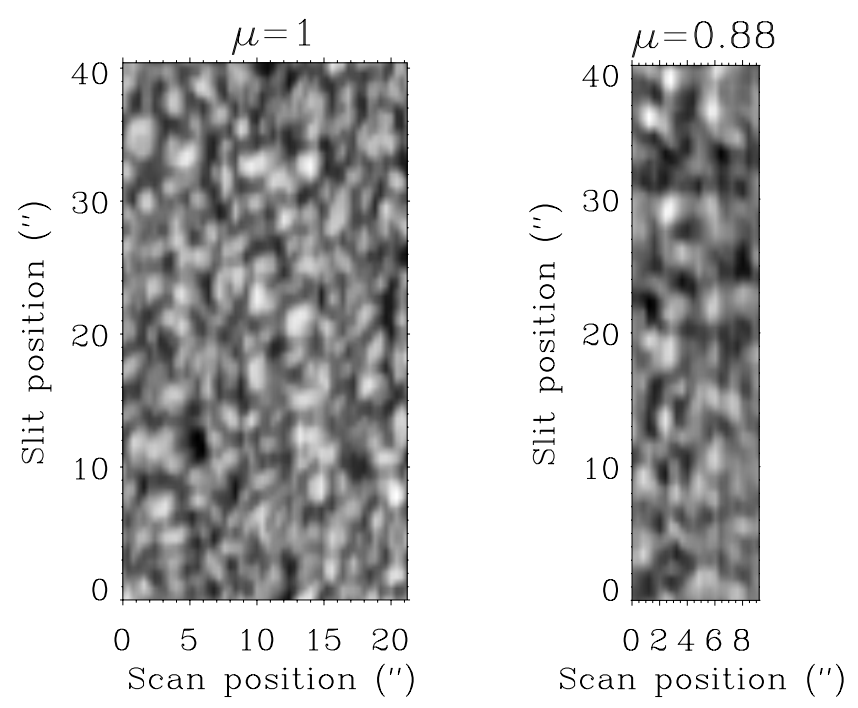

Fig. 1. Continuum maps of the observations taken at disc centre (left) and at $\mu=0.88$ (right).

The standard reduction of the data included dark current and flatfield corrections. The polarimetric calibration was performed with the aid of polarization optics of known properties, located at an appropriate place in the optical path. Any residual crosstalk between the Stokes parameters, because of the uncalibrated coelostat mirrors, were removed using statistical techniques described in Collados (2003). After that, a statistical procedure called Principal Component Analysis (PCA) was applied to reduce the noise level of the polarization profiles. This consists of creating a basis with the eigenvectors of the correlation matrix of the observations in which they can be represented. Then we can eliminate from the basis those eigenprofiles that have no information about the real signals and are dominated by noise and reconstruct the observations with this shortened base. This is feasible because of the fast decrease of the eigenvalues and allows us to increase the noise level of the observations.

After denoising with PCA, the noise level of Stokes $V$ is $6.5 \times 10^{-5} I_{\mathrm{c}}$ and $9.0 \times 10^{-5} I_{\mathrm{c}}$ for the observations at $\mu=1$ and $\mu=0.88$, respectively. Stokes $Q$ has a noise level of $1.1 \times$ $10^{-4} I_{\mathrm{c}}$ and $8.5 \times 10^{-5} I_{\mathrm{c}}$ and Stokes $U 2.0 \times 10^{-4} I_{\mathrm{c}}$ and $5.0 \times$ $10^{-5} I_{\mathrm{c}}$ at disc centre and at $\mu=0.88$, respectively.

\section{Data analysis}

We obtain the magnetic field vector by inverting the four Stokes profiles using the $\mathrm{SIR}^{1}$ code (Ruiz Cobo \& del Toro Iniesta 1992). We perform a two component inversion based on a magnetic atmosphere that occupies a fraction of the resolution element and a field-free one filling the rest of the space. Including two components in the inversion increases the number of free parameters. Then, in order to have an adequate number of degrees of freedom (see Asensio Ramos et al. 2007b) we chose the majority of the variables to be constant with height. The microturbulent velocity in both components, the line of sight flow velocity of the magnetic component, the magnetic field strength and the azimuth of the magnetic field vector are assumed to be constant with height. The macroturbulent velocity is forced to be the same in both atmospheres. The macroscopic velocity of the non magnetic atmosphere has 3 nodes and the inclination of

\footnotetext{
1 Stokes Inversion based on Response functions.
}

the magnetic field vector has 2 nodes. The temperature stratification of each atmosphere was allowed to vary independently with 5 nodes.

In Fig. 2 we show the maps of the amplitude of circular polarization for both scans. Superimposed on them, some contours of equal amplitude of linear polarization $\left(\sqrt{A_{Q}^{2}+A_{U}^{2}}\right)$ are plotted ( $A_{Q}$ and $A_{U}$ represent the maximum absolute amplitude of the Stokes $Q$ and $U$ profiles). In both maps there are numerous examples of a linear polarization signal lying in between two opposite polarity circular polarization signals. Small lowlying magnetic loops are expected to show just such a signature: the magnetic field vector points up on one side of the structure, is horizontal in the middle and points down on the other side. We found 6 clear cases of such structures in the map at disc centre and 5 in the map at $\mu=0.88$. They are enclosed by the white rectangles in Fig. 2. In the examples in the $\mu=0.88$ map all the linear polarization is slightly displaced towards one of the circular polarization signals. In the disc centre map this is not a systematic behaviour. It might be a projection effect or that the foot points have different field strengths.

We now discuss in detail the results from inversions of the clearest loop identified by the thick dashed-line rectangle in the observation at $\mu=0.88$ (the others show similar properties and geometries). The inclination and azimuth of the magnetic field vector recovered from the inversions in the line-of-sight reference frame are transformed to the local reference frame (without solving the $180^{\circ}$ degree azimuth ambiguity).

In the left panel of Fig. 3, the horizontal component of the magnetic flux vector is presented together with the vertical magnetic flux density for the thick dashed rectangle in Fig. 2. The white lines in the left panel of Fig. 3 represent the direction of the magnetic field vector. The values of the background flux density clearly correspond to internetwork regions in all the pixels containing the loop-like structure. The horizontal component of the magnetic field vector in the pixels in between the two opposite polarities is approximately directed along the line that joins them. In the central and right panels of Fig. 3, we plot the magnetic field lines on a vertical cut through the atmosphere along the line joining the two polarities. The vertical magnetic flux density is indicated by the colour scale and the superimposed arrows represent the magnetic field vectors. The azimuth values differ by $180^{\circ}$ in the two panels, both solutions being consistent with the observed spectral profiles. Note that the inclination of the magnetic field vector is nearly height independent even if we allow it to vary. The magnetic field lines have been computed as those lines that are parallel to the vector field. In each slit position in the centre and right panels the geometrical heights have been displaced to have the same gas pressure at $h=0$. The reference gas pressure value is the mean value at $h=0$. The applied displacements are small, having a mean value of $20.5 \mathrm{~km}$. The $\Omega$-loop (centre) or $U$-loop (right) structure is evident: the magnetic field is almost vertical at the points where the strongest Stokes $V$ signals are (at the loop foot points) and is horizontal in the transition between one polarity and the other. Recently López Ariste et al. (2006a) have found $U$-loops in the photosphere below a filament structure by solving the $180^{\circ}$ ambiguity inherent to the Zeeman effect, suggesting that these structures are at least allowed by the physics that drives the solar atmosphere. In our case, unfortunately, we do not have any observational constraint to choose between the two solutions shown in Fig. 3.

There are many bipolar regions potentially harboring lowlying loops in the maps in Fig. 2 but only those boxed in are as 

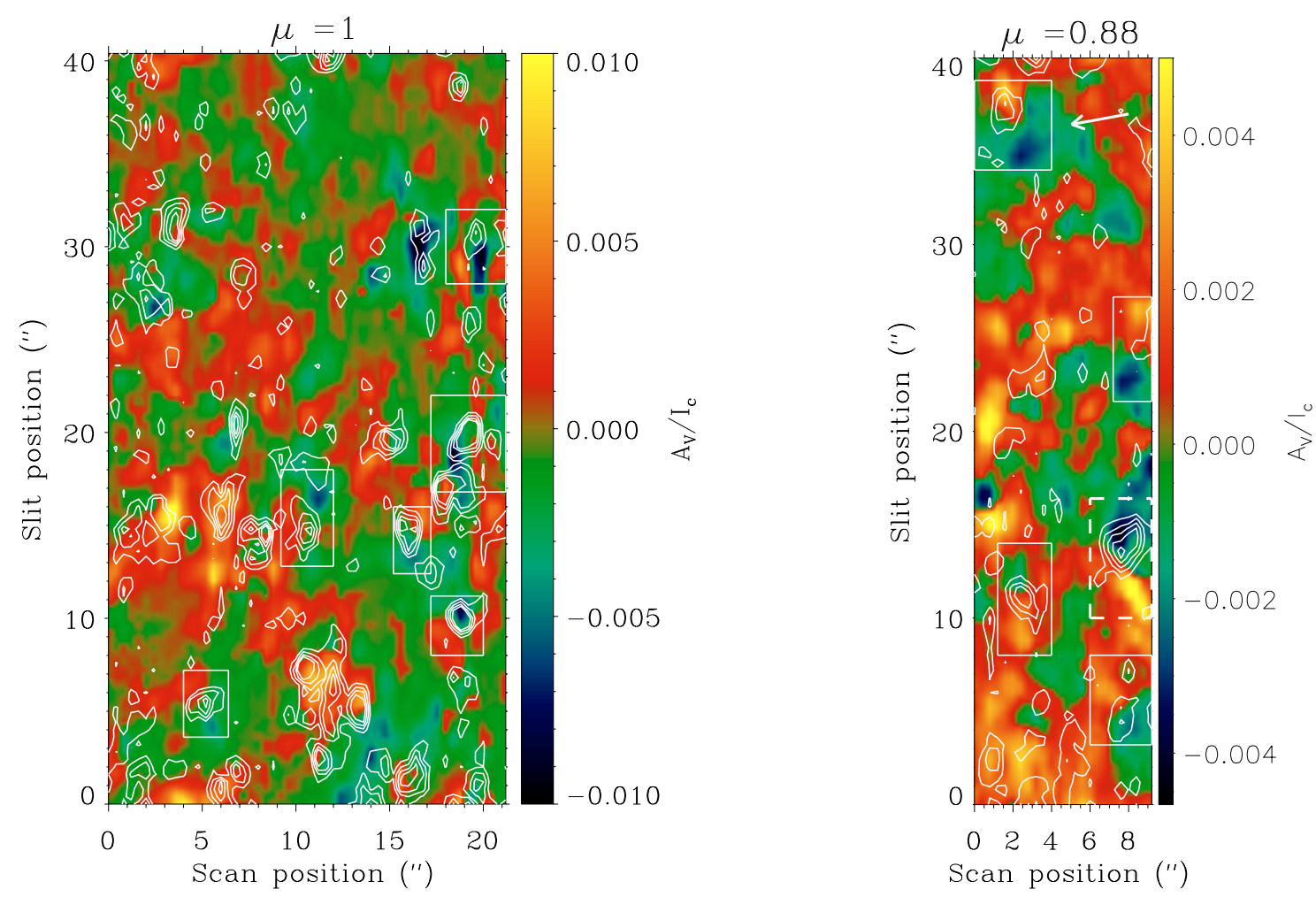

Fig. 2. Maps of the amplitude $A_{\mathrm{V}}$ of circular polarization (colour scale) of the observations taken at disc centre (left) and $\mu=0.88$ (right). The white contours represent the degree of linear polarization $\sqrt{A_{Q}^{2}+A_{U}^{2}}$ having the following values: $1.5 \times 10^{-3}, 2 \times 10^{-3}, 2.5 \times 10^{-3}, 3 \times 10^{-3} I_{\mathrm{c}}$ for the map at disc centre and additionally at $1 \times 10^{-3}$ for the map at $\mu=0.88$. The white arrow in the $\mu=0.88$ map points towards the position of the disc centre.
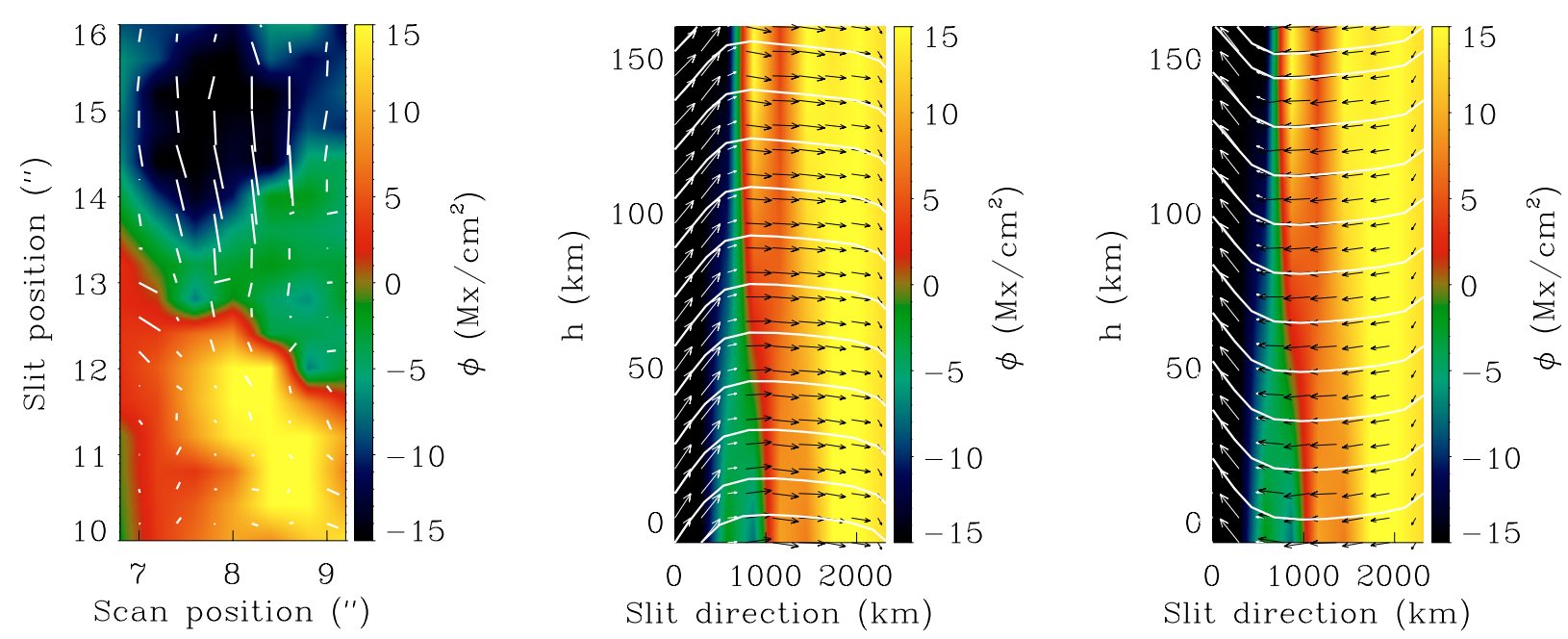

Fig. 3. Left panel: map of the vertical magnetic flux density of the loop selected as an example (marked with a dashed square in the $\mu=0.88$ circular polarization map). The white lines represent the horizontal component of the magnetic flux vector. Centre and right panels: magnetic field lines computed for the two allowed magnetic field configurations, that differ by $180^{\circ}$ in azimuth, giving rise to an $\Omega$ - and a $U$-loop structure, respectively. The inclination and azimuth of the magnetic field vector have been translated to the local reference system.

clear as the example shown in Fig. 3. This means that in all these cases the horizontal component of the magnetic vector is mainly directed along the line joining the opposite magnetic polarities and that the inclination is almost vertical in the foot points of the structure and rather horizontal in between. The observed loops are rather flat, with a foot point separation of $2300 \mathrm{~km}$ and with at least some of the field lines at the loop top lying in the height range of formation of the $1.56 \mu \mathrm{m}$ lines (i.e. only a few hundred $\mathrm{km}$ above the foot points). All cases also share some other properties. The magnetic field strength is weak, being below $500 \mathrm{G}$ in the majority of the cases. There are only very few points reaching higher values such $800-1000 \mathrm{G}$.

The line of sight plasma flows in the magnetic atmosphere are correlated with the continuum intensity: bright structures in the continuum image are associated with upflows in the magnetic component, dark structures with downflows. The foot points of the magnetic structures are located equally probably in upflows or in downflows as seen in Fig. 4. The more vertical fields are 


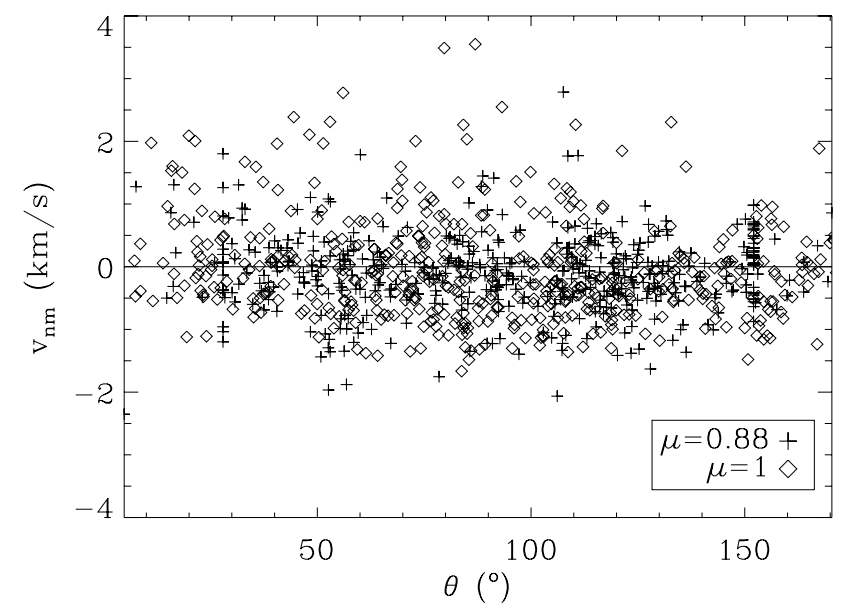

Fig. 4. Line-of-sight bulk velocity of the field free component plotted versus the inclination of the magnetic field inclination with respect to the local vertical. Positive values correspond to downflows and negatives to upflows.

located at the footpoints of the structure and we can see that they are found either in upflows or downflows. Also the more horizontal fields lie both in upflows and downflows. This could be indicative of a dynamic scenario. If the time scale of the evolution of such structures is longer than the convective turnover time of granulation, one would expect $\Omega$-loops to be anchored in intergranular lanes (where downflows dominate) and to be lying over granules. For $U$-loops the situation is expected to be reversed, however. Consequently, we may be seeing a mixture of $\Omega$ and $U$-loops.

As seen in Fig. 3, the magnetic flux density of the selected loop has values typical of the internetwork. This is also true for the rest of the loops boxed in Fig. 2. The magnetic flux density in the pixels occupied by loops follows the same distribution as the pixels in the rest of the internetwork area. Also the magnetic field strength distribution is the same as that of the rest of the area covered by the typical internetwork. The fact that both the selected loops and the rest of the observed area share the same properties suggests that the internetwork could harbour many of these small low-lying loops, most of them remaining undetectable in our observations.

\section{Discussion and conclusions}

We have detected 11 examples of low-lying (i.e. photospheric) loops in two scans of internetwork regions in the quiet Sun. These loops may be related to the horizontal features observed by Lites et al. (1996) and also the emerging flux in form of small bipoles observed by de Pontieu (2002) using the visible lines at $630 \mathrm{~nm}$. In our case, we have more sensitive spectropolarimetric observations (very magnetically sensitive infrared lines and very low noise in both linear and circular polarization) which allow us to reconstruct the topology of the magnetic structures. Although we could not follow the evolution of such loop-like structures, one may speculate that the physical processes that give rise to these loops are small scale magnetic flux emergences in the internetwork quiet Sun.

Concerning the magnetic flux carried by the loop-like structures that we have identified, we find that at least $6.1 \%$ (disc centre) and $25.8 \%(\mu=0.88)$ of the magnetic flux in the whole map emerges in the form of small-scale magnetic loops. On average, this means that at least $10-20 \%$ of the magnetic flux in the solar internetwork is in the form of low-lying loops at any given time. This is a lower limit since such features may have escaped detection due to the weakness of the associated linear polarization signals. One implication of the fact that these loops are so low-lying is that the magnetic flux they are connecting is unlikely to reach the chromosphere and higher layers. Unless we are observing a transient phase, this implies that possibly much of the magnetic flux does not rise above the photosphere. This point deserves further attention in view of the suggestions of Trujillo Bueno et al. (2004) that a tangled field could provide a clue to understanding how the solar chromosphere and corona are heated.

Acknowledgements. This research has been funded by the Spanish Ministerio de Educación y Ciencia through project AYA2004-05792. Part of this work was done when M. J. Martínez González was visiting the Max Planck Institute in Lindau and she would like to express her gratitude to the staff for their warm hospitality. The authors acknowledge the comments of the anonymous referee which helped to improve the letter and strengthen the conclusions.

\section{References}

Asensio Ramos, A., Martínez González, M. J., López Ariste, A., Trujillo Bueno, J., \& Collados, M. 2007a, ApJ, in press

Asensio Ramos, A., Socas-Navarro, H., López Ariste, A., \& Martínez González, M. J. 2007b, ApJ, in press

Ballesteros, E., Collados, M., Bonet, J. A., et al. 1996, A\&A, 115, 353

Collados, M. 1999, in Third Advances in Solar Physics Euroconference, ed. B. Schmieder, A. Hofmann, \& J. Staude, ASP Conf., 184, 3

Collados, M. V. 2003, in Polarimetry in Astronomy, ed. S. Fineschi, Proc. SPIE, 4843, 55

de Pontieu, B. 2002, ApJ, 569, 474

Faurobert, M., Arnaud, J., Vigneau, J., \& Frisch, H. 2001, A\&A, 378, 267

Feldman, U., Widing, K. G., \& Warren, H. P. 1999, ApJ, 522, 1133

Keller, C. U., Deubner, F., Egger, U., Fleck, B., \& Povel, H. 1994, A\&A, 286, 626

Khomenko, E. V., Collados, M., Solanki, S. K., Lagg, A., \& Trujillo Bueno, J. 2003, A\&A, 408, 1115

Lin, H. 1995, ApJ, 446, 421

Lin, H., \& Rimmele, T. 1999, ApJ, 514, 448

Lites, B. W., Leka, K. D., Skumanich, A. P., Martínez Pillet, V., \& Shimizu, T. 1996, ApJ, 460, 1019

López Ariste, A., Aulanier, G., Schmieder, B., \& Sainz Dalda, A. 2006a, A\&A, 456,725

López Ariste, A., Tomczyk, S., \& Casini, R. 2006b, A\&A, 454, 663

Manso Sainz, R., Landi Degl' Innocenti, E., \& Trujillo Bueno, J. 2004, ApJ, 614, $89 \mathrm{~L}$

Martínez González, M. J., Collados, M., \& Ruiz Cobo, B. 2007, in 4th Solar Polarization Workshop, ed. R. Casini, \& B. W. Lites, ASP Conf. Ser., in press Ruiz Cobo, B., \& del Toro Iniesta, J. C. 1992, ApJ, 398, 375

Schmidt, W., \& Kentischer, T. 1995, A\&A, 113, 363

Solanki, S. K., Lagg, A., Woch, J., Krupp, N., \& Collados, M. 2003, Nature, 425, 692

Stenflo, J. O. 1987, Sol. Phys., 114, 1

Trujillo Bueno, J., Shchukina, N., \& Asensio Ramos, A. 2004, Nature, 430, 326 